\title{
Effects of acupuncture on quality of life and pain in patients with osteoporosis-a pilot randomized controlled trial: reply to comments by Moran et al.
}

\author{
Joerg Schiller ${ }^{1}$
}

Received: 14 December 2016 / Accepted: 15 December 2016 /Published online: 10 January 2017

(C) International Osteoporosis Foundation and National Osteoporosis Foundation 2016

Dear editor,

We appreciate the interest of Moran et al. in our article [1] and their critical impulse regarding the sample size calculation [2]. In our paper, we present the original sample size calculation as done for the study protocol. This calculation of the number of cases is based on the work of Inoue [3] and Itou [4]. However, according to the pilot character of the study after the end of the intervention phase, we revised our strategy for data analyses based on the large number of measurements over time and the additional 3-month follow-up. Mixed models are more appropriate and have more statistical power when analyzing datasets with repeated measurements. We agree that the paper of Guo et al. [5] presents a reasonable way to calculate the sample size when using mixed models. The mentioned references by Kanis et al. [6,7] have been cited in the introduction of the manuscript and do not relate to the sample size calculation. These references are obviously placed incorrectly according to our continuous revision of the manuscript. Thanks for this very helpful note, the faulty citation is very unpleasant for us and we would like to correct this herewith. The correct citations in the section "Statistical analysis" are the ones mentioned above by Inoue [3] and Itou [4].

Joerg Schiller

Schiller.Joerg@mh-hannover.de

1 Department of Rehabilitation Medicine, Hannover Medical School, Carl-Neuberg-Str. 1, 30655 Hannover, Germany
Compliance with ethical standards

Conflicts of interest None.

\section{References}

1. Schiller J, Korallus C, Bethge M, Karst M, Schmalhnofer ML, Gutenbrunner C, Fink MG (2016) Effects of acupuncture on quality of life and pain in patients with osteoporosis - a pilot randomized controlled trial. Arch Osteoporos 11:34. doi:10.1007/s11657-0160288-X

2. Jose M. Moran JM, Ignacio Aliaga I, Pedrera-Zamorano JD (2016) The importance of accurate sample size calculations in clinical trials. Arch Osteoporos 12. doi: 10.1007/s11657-016-0304-1

3. Inoue M, Kitakoji H, Ishizaki N, Tawa M, Yano T, Katsumi Y, Kawakita K (2006) Relief of low back pain immediately after acupuncture treatment - a randomised, placebo controlled trial. Acupunct Med 24(3):103-108

4. Itoh K, Katsumi Y, Hirota S, Kitakoji H (2006) Effects of trigger point acupuncture on chronic low back pain in elderly patients - a sham-controlled randomised trial. Acupunct Med 24(1):5-12

5. Guo Y, Logan HL, Glueck DH, Muller KE (2013) Selecting a sample size for studies with repeated measures. BMC Med Res Methodol

6. Kanis JA, Oden A, Johnell O, De Laet C, Jonsson B (2004) Excess mortality after hospitalisation for vertebral fracture. Osteoporos Int 15:108-112

7. Kanis JA, Johnell O, Oden A, Sembo I, Redlund-Johnell I, Dawson A, De Laet C, Jonsson B (2000) Long-term risk of osteoporotic fracture in Malmo. Osteoporos Int 11:669-674 